\section{Total lung collapse after pulmonary infarction}

Soror Aithan, Javed Khan, Maher Bazarbashi

\begin{abstract}
A case is presented of total lung collapse secondary to intrabronchial blood clot as a complication of pulmonary infarction. The blood clot was removed at bronchoscopy with complete re-expansion of the lung.
\end{abstract}

(Thorax 1994;49:938-939)

Total lung collapse due to pulmonary infarction is a rare and unusual condition. ${ }^{1}$ It may result from obstruction of the bronchus secondary to intrabronchial blood clot. We present a case of total lung collapse which occurred following pulmonary infarction.

\section{Case report}

A 36 year old woman at 24 weeks gestation presented with frank haemoptysis. Two weeks before presentation she had noted progressive exertional dyspnoea.

Examination revealed a blood pressure of $110 / 80$, pulse 90 beats/minute regular, and respiration of 24 breaths/minute. On auscultation wheezes were noted on the right side of the chest; cardiovascular examination was normal. A chest radiograph revealed an elevated right hemidiaphragm and clear lung fields. Based on a clinical diagnosis of pulmonary embolism the patient commenced intravenous heparin therapy.

Twelve hours later she developed sudden severe right sided pleuritic chest pain with further haemoptysis and dyspnoea, her respiratory rate increased to 40 breaths/minute and pulse rate increased to 130 beats/minute while her blood pressure remained stable at 110/80.

Clinical examination revealed no cyanosis but there was dullness and diminished breath sounds in the right chest with a shift of the trachea to the right. The ECG was normal and arterial blood gas tension whilst breathing air showed hypoxaemia. A chest radiograph showed complete collapse of the right lung with loss of airway contours distal to the carina (fig 1). Heparin was continued in therapeutic doses.

The patient was transferred to our hospital. Additional history revealed a 12-hour car journey three weeks prior to this presentation, but no other risk factor or previous history of thromboembolic disease was noted. She had had three previous normal and uncomplicated pregnancies.

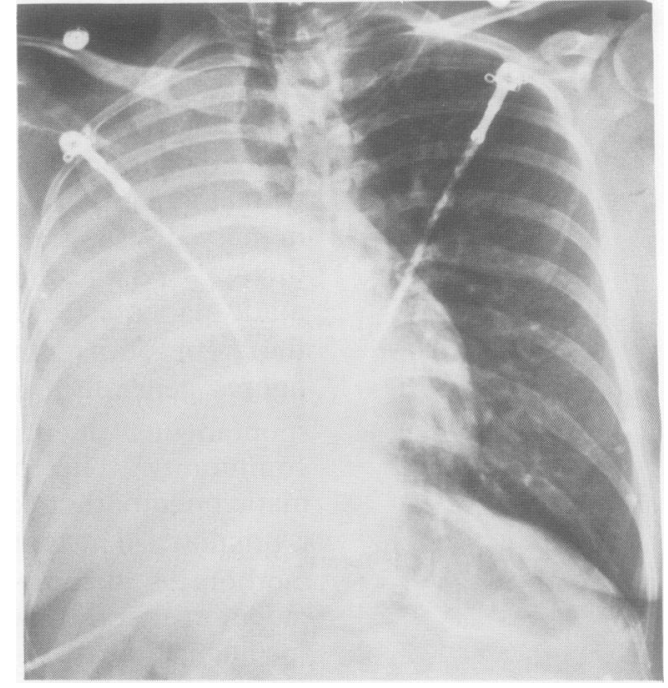

Figure 1 Chest radiograph showing collapse of right lung with mediastinal shift.

On arrival examination revealed an acutely ill woman in moderate respiratory distress. Her chest examination was consistent with right lung collapse, there was no clinical evidence of a deep venous thrombosis, and the radiograph was unchanged. Laboratory tests showed a normal blood count with partial thromboplastin time 52.6 (26-39) and prothrombin time 13 (11-14). Arterial blood gas tensions on room air were $\mathrm{pH} 7 \cdot 45, \mathrm{PCO}_{2} 3.53 \mathrm{kPa}, \mathrm{PO}_{2} 7 \cdot 35 \mathrm{kPa}$, SAT $91 \%$.

Selective right pulmonary angiography revealed a cut off of blood flow to the right lower lobe and no active bleeding was noted (fig 2). Fibreoptic bronchoscopy showed complete obstruction of the right main bronchus due to blood clot which was partially removed with biopsy forceps and suction. A chest radiograph after bronchoscopy showed re-expansion of the right upper lobe. A second fibreoptic bronchoscopic examination was performed on the third hospital day and attempts to remove the clots

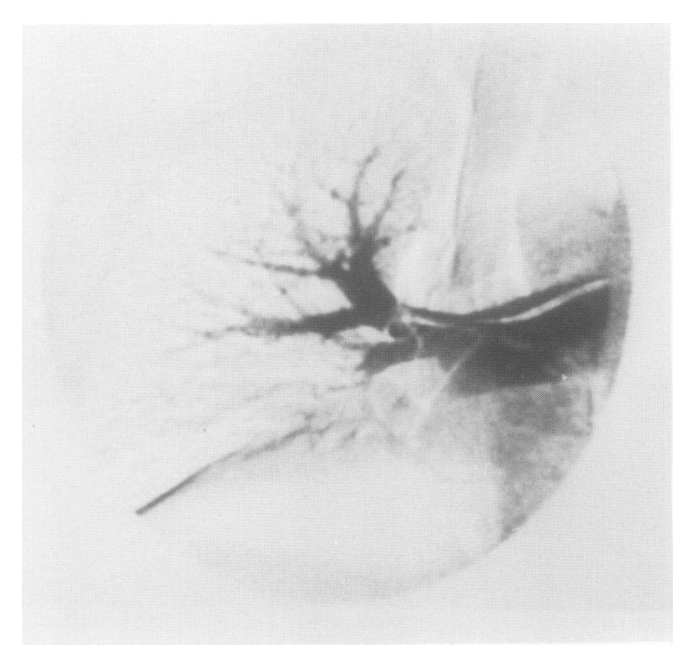

Figure 2 Pulmonary angiographic scan showing no flow in right lower lobe artery. 
with suction and forceps were successful in reexpanding the right middle lobe. Because of the persistent collapse of the right lower lobe, further examination by rigid bronchoscopy was performed and all visible clots were removed. Intravenous heparin was continued.

A subsequent chest radiograph showed complete expansion of the right lung with elevation of the right hemidiaphragm.

The patient was then discharged on subcutaneous heparin 10000 units every eight hours. At three months follow up the patient remained well. Sixteen weeks later she delivered a normal healthy baby.

\section{Discussion}

Only one previous report of total lung collapse due to pulmonary infarction has been found in the literature ${ }^{1}$ although seven cases of bronchial bleeding due to heparin have been described. ${ }^{4}{ }^{7}$

As with the case reported in the literature, our patient also developed haemoptysis before starting heparin. It is very likely that heparin therapy may have increased the bleeding that caused the clot in the bronchial tree. ${ }^{6}$ In the presence of total lung collapse ventilation-perfusion scanning is unhelpful, ${ }^{8}$ so pulmonary angiography was performed which could confirm the diagnosis and may also demonstrate a bleeding vessel.

The patient showed clinical and radiological improvement after removal of the blood clot through flexible and rigid bronchoscopy, whereas the other reported patient required a period of intubation and mechanical ventilation.
Fibreoptic bronchoscopy has been used in the removal of foreign bodies. Blood clot removal has also been reported in emergency cases using a Fogarty balloon tip embolectomy catheter through a flexible fibreoptic bronchoscope. ${ }^{9}$ The fact that three attempts were required to complete the bronchial toilet suggests that probably the initial use of a rigid instrument would have been preferable.

The use of heparin therapy in such situations is controversial. It has been suggested that heparin should not be used until bleeding stops. ${ }^{\prime}$ However, we continued heparin therapy with a successful outcome.

We suggest that pulmonary infarction should be considered in the differential diagnosis of total lung collapse when it is associated with haemoptysis.

1 Van Renterghem D, Bogaerts Y, Tasson J, Van Der Stracton. Intrabronchial bleeding and life-threatening atelectasis in pulmonary embolism. Eur f Respir Dis 1984;65:144-6.

2 Stein PD, Terrin ML, Hales CA, Palevsky HI, Saltzman HA, Thompson T, et al. Clinical, laboratory, roentgenographic and electrocardiographic findings in patients with acute embolism and no pre-existing cardiac or pulmonary disease. Chest 1991;100:598-603.

3 Stein PD, Gotischalk A, Saltzman HA, Terrin ML. Diagnosis of acute pulmonary embolism in the elderly: $f \mathrm{Am} \mathrm{Coll}$ of acute pulmonary embol

4 Bone RS, Jay SJ, Reynolds RC, Johanson WG. Massive pulmonary hemorrhage: a rare complication of heparin therapy. Am f Med Sci 1976;272:297-9.

5 Ferris EJ. Pulmonary hemorrhage. Vascular evaluation and interventional therapy. Chest 1981;80:710-4

6 Jick H, Slone D, Borda IT, Shapiro S. Efficacy and toxicity of heparin in relation to age and sex. $N$ Engl $\mathcal{F}$ Mid 1968 ; 279:284-6.

7 Kernohan RJ, Todd C. Heparin therapy in thrombo-embolic disease. Lancet 1966;438:621-3.

8 Chung CJ, Grossnickle M, Rosenthal P, Hartly S, Gordon L. Postatelectatic ventilation-perfusion mismatch simulating a postatelectatic ventilation-perfusion mismatch simula

9 Allen AR, Siefkin AD. Emergency airway clot removal in acute hemorrhagic respiratory failure. Crit Car Med 1987; 15:985. 